\title{
Immunological memory to Common Cold Coronaviruses assessed longitudinally over a three-year period
}

\author{
Esther Dawen $\mathrm{Yu}^{1}$, Tara M. Narowski ${ }^{2}$, Eric Wang ${ }^{1}$, Emily Garrigan ${ }^{1}$, Jose Mateus ${ }^{1}$, April \\ Frazier $^{1}$, Daniela Weiskopf ${ }^{1}$, Alba Grifoni ${ }^{1}$, Lakshmanane Premkumar $^{2}$, Ricardo da Silva \\ Antunes ${ }^{1, \#, *}$, and Alessandro Sette ${ }^{1,3, \#, *}$ \\ ${ }^{1}$ Center for Infectious Disease and Vaccine Research, La Jolla Institute for Immunology (LJI), La Jolla, CA 92037, \\ USA \\ ${ }^{2}$ Department of Microbiology and Immunology, University of North Carolina School of Medicine, Chapel Hill, NC \\ 27599-7290, USA. \\ ${ }^{3}$ Department of Medicine, Division of Infectious Diseases and Global Public Health, University of California, San \\ Diego (UCSD), La Jolla, CA 92037, USA \\ \# These authors contributed equally \\ * Correspondence: rantunes@liji.org (R.d.S.A.), alex@liji.org (A.S.)
}




\begin{abstract}
Understanding immune memory to Common Cold Coronaviruses (CCCs) is relevant for assessing its potential impact on the outcomes of SARS-CoV-2 infection, and for the prospects of pan-corona vaccines development. We performed a longitudinal analysis, of pre-pandemic samples collected from 2016-2019. CD4+ T cells and antibody responses specific for CCC and to other respiratory viruses, and chronic or ubiquitous pathogens were assessed. CCC-specific memory CD4+ T cells were detected in most subjects, and their frequencies were comparable to those for other common antigens. Notably, responses to CCC and other antigens such as influenza and Tetanus Toxoid (TT) were sustained over time. CCC-specific CD4+ T cell responses were also associated with low numbers of HLA-DR+CD38+ cells and their magnitude did not correlate with yearly changes in the prevalence of CCC infections. Similarly, spike RBD-specific IgG responses for CCC were stable throughout the sampling period. Finally, high CD4+ T cell reactivity to $\mathrm{CCC}$, but not antibody responses, was associated with high pre-existing SARS-CoV2 immunity. Overall, these results suggest that the steady and sustained CCC responses observed in the study cohort are likely due to a relatively stable pool of CCC-specific memory CD4+ T cells instead of fast decaying responses and frequent reinfections.
\end{abstract}




\section{Introduction}

Common Cold Coronaviruses (CCCs) are seasonal viruses comprising of two subtypes, namely $\alpha$-coronaviruses (HCoV-229E and HCoV-NL63) and $\beta$-coronaviruses (HCoV-OC43 and HCoV-HKU1), that most frequently cause mild illnesses in humans ${ }^{1-5}$. CCC are endemic viruses with widespread global distribution and have long circulated in humans. These CCC viruses are phylogenetically related to other coronaviruses that cause severe disease in humans, such as SARS-CoV-2, SARS-CoV and MERS-CoV ${ }^{6}$.

CCC have been estimated to be responsible for up to $15-30 \%$ of pre-pandemic annual respiratory tract infections ${ }^{7-9}$, with infections occurring most frequently in young children ${ }^{7,10,11}$. CCC infections are associated with a clear seasonality, but infection can occur at any time of the year $^{12-15}$.

Whether immunity to CCC viruses is short or long lived has been debated with conflicting reports ${ }^{16-22}$. Some discrepancies may be reconciled as some reports consider immunity as protection from re-infection while others consider protection from symptomatic disease. CCC infections are associated with generation of antibody titers widely detectable in the human population ${ }^{17,23-25}$. However, little data is available regarding the frequency of memory $\mathrm{T}$ cell responses against $\mathrm{CCC}$, and in particular their stability overtime. Understanding the steady state dynamics of CCC antibody and T cell responses in humans is of potential relevance in the context of the long-term evolution of the SARS-CoV-2 pandemic, in the context of the current scenario, where a large fraction of the human population is exposed and/or vaccinated.

Furthermore, it has been widely reported that CCC $\mathrm{T}$ cell responses are associated with some degree of cross-reactivity with SARS-CoV-2, and that this cross-reactivity can at least in part explain the pre-existing $\mathrm{T}$ cell memory reactivity recognizing SARS-CoV-2 sequences, observed in SARS-CoV-2 unexposed subjects ${ }^{25-29}$. A putative role for CCC cross-reactive T cells in modulating COVID-19 vaccination and disease outcomes has been indicated by several independent studies ${ }^{26,30-33}$. However, it is not clearly understood which factors in a given population, determine which individuals are associated with pre-existing SARS-CoV-2 T cell memory reactivity. Understanding the dynamics of CCC cross-reactivity with SARS-CoV-2 T cell responses is of potential relevance for understanding variations in COVID-19 disease severity, and also relevant to the potential development of pan-corona $T$ cell vaccination ${ }^{34}$. 
Herein we performed a longitudinal analysis, over the course of 6 months up to 3 years, of $\mathrm{CD} 4+\mathrm{T}$ cells and antibody responses to $\mathrm{CCC}$ and responses to other respiratory viruses, and chronic or ubiquitous pathogens. Overall, the results suggest that responses are readily detectable, and sustained over time. 


\section{Results}

\section{Frequency of $\mathrm{CCC}$-specific memory $\mathrm{CD}^{+} \mathrm{T}$ cells are comparable to those for other common antigens}

We studied PBMC samples from 32 participants of a Bordetella pertussis observational study ${ }^{35}$. Three to seven longitudinal blood donations per donor, spanning time periods from 6 months to more than 3 years were available. All samples were collected in the 2016 to 2019 period (pre-pandemic). Subjects (9 male, 23 female) represented a range of ethnicities (14 Caucasian, 10 Hispanics, 7 Asian and 1 Black), with a median age of 24.5 years (range 18-35) (Table 1), and were recruited at LJI (La Jolla CA).

CD4+ T cell responses to the four prototypical CCC viruses (NL63, 229E, HKU1 and OC43) were measured, using the Activation Induced Marker (AIM) and the OX40/4-1BB markers combination $^{26}$, which has been previously utilized to characterize viral responses and particularly SARS-CoV-2 CD4+ T cell responses ${ }^{36-40}$. Responses to other respiratory viruses (influenza, RSV, and rhinovirus), chronically infectious viruses (EBV, CMV, and VZV), and ubiquitous bacterial vaccine antigens (TT and PT) were measured using specific peptide sets (Table 2 and methods section). $\mathrm{CD}^{+} \mathrm{T}$ cell responses were measured in the 32 study subjects at the first-time point of the longitudinal series (Fig. 1). Significant antigen specific CD4+ T cell responses were detected for all four CCC epitope pools. Overall, 81.3\%, 75.0\%, 71.9\%, and 78.1\% of the donors were positive for NL63, 229E, HKU1 and OC43, respectively; median magnitude of CD4+ T cell responses was $0.089 \%, 0.083 \%, 0.078 \%$, and $0.077 \%$ for NL63, 229E, HKU1 and OC43, respectively (Fig. 1). Similar reactivity across the $4 \mathrm{CCC}$ were observed when considering SI responses $\geq 2$ (Fig. S1) consistent with previous observations ${ }^{26}$.

The CCC-specific CD4+ T cell reactivities were in the same range as those detected for the RSV, CMV, EBV, VZV and PT targets (Fig. 1). CCC-specific CD4+ T cell reactivities were 2-3-fold lower than Flu ( $\mathrm{p}$ values ranging 0.0003-0.003 and $\mathrm{p}=0.01-0.04$ for absolute and SI readouts, respectively) or TT ( $\mathrm{p}$ values ranging $0.017-0.04$ and $\mathrm{p}=0.003-0.004$ for absolute and SI readouts, respectively) responses, and 2-fold higher compared to rhinovirus response ( $\mathrm{p}$ values ranging 0.014-0.047 and $\mathrm{p}=0.024-0.036$ for absolute and SI readouts, respectively) (Fig. 1 and S1).

As expected, CCC specific CD4+ T cells predominantly correspond to TCM and TEM memory compartments (defined as CD45RA-CCR7+ and CD45RA-CCR7-, respectively), with 
minimal contributions of naïve (CD45RA+CCR7+) or TEMRA (CD45RA+CCR7-) compartments (Fig. 2). Similar phenotypes were associated with the AIM+ CD4+ T cells responding to the other antigen targets (data not shown). In summary, the data demonstrates that CD4+ T cell reactivity to 229E, NL63, HKU1 and OC43 was frequently detected in the study cohort, mediated by classic conventional memory cells and in the same order of magnitude as other viral antigens.

\section{Longitudinal analysis of CD4+ $\mathrm{T}$ cell reactivity to $\mathrm{CCC}$ and other antigens}

We performed a longitudinal analysis of the levels of CD4+ T cell responses to CCC and other antigens. For each antigen, half-lives $\left(\mathrm{t} \frac{1}{2}\right)$ were calculated based on linear mixed effects models using $\mathrm{R}$ package nlme ${ }^{41}$, analyzing longitudinal responses for each individual. As shown in Fig. 3A, CCC CD4+ T cell responses were essentially steady over time (t $1 / 2$ ranging to 244 years to no decline). Similarly, sustained responses were observed for the other antigenic targets (Fig. 3B). In particular, no decline was observed in the case of EBV, TT and PT or a modest decline ( $\mathrm{t} 1 / 2=7.4$ years) in the case of influenza (Fig. 3C). Comparable patterns were observed for RSV, rhinovirus, CMV and VZV (Fig. 3B). Overall, these results indicate relatively constant and stable responses to $\mathrm{CCC}$ over the time considered, and in line to what observed for other antigens.

To gain more insight in whether these apparently stable responses originate from frequent reinfections or long-lasting durable responses we determined the range of fluctuation of CD4+ T cell responses. This was done by first normalizing responses for each donor and each antigen, and then calculating the associated 5th-95th percentile range. We expected that responses to influenza, where yearly vaccination/exposures are relatively common, would fluctuate more than responses to other antigens, such as TT, for which natural exposure and re-vaccination is expected to be less frequent. The data in Fig. 4A indicates that this is indeed the case. Importantly, the patterns of fluctuation of CD4+ T cell responses, more specifically the $5^{\text {th }}-95^{\text {th }}$ percentile range for each CCC viruses (ranging 0.31-3.85) were similar to that observed for TT (0.35-2.6), and lower than what was observed in the case of influenza (0.06-2.96). These data further indicated durable and constant CD4+ $\mathrm{T}$ cell responses to $\mathrm{CCC}$ overtime. 


\section{HLA-DR ${ }^{+}$CD38+ expression and periodicity of $\mathrm{CCC}$ antigen-specific CD4+ $\mathrm{T}$ cells}

The expression of the HLA-DR and CD38 markers is associated with recent in vivo activation ${ }^{26,42,43}$. Fig. 4B indicate that CD4+ T cells responding to the CCC peptides are associated with a 3.7-3.9\% range of HLA-DR ${ }^{+} \mathrm{CD} 38^{+} \mathrm{AIM}+\mathrm{CD} 4+\mathrm{T}$ cells $(95 \%$ confidence interval of $0.2-$ 9.6\%). Only few data points were above a threshold of high reactivity $(>10 \%)$, which we previously associated with recent infection by SARS-CoV-2 of a cohort of COVID-19 convalescent subjects ${ }^{26}$. Interestingly, influenza-specific CD4+ T cells were associated with a median of HLA-DR ${ }^{+} \mathrm{CD} 38^{+} \mathrm{AIM}+\mathrm{CD} 4+\mathrm{T}$ cells of $7.8 \%$ (95\% confidence interval of 2.2-16.0\%), and $30 \%$ of the data points (donor/time point instances) were associated with values of $10 \%$ or higher. In the case of TT, the percent of $\mathrm{HLA}_{-} \mathrm{DR}^{+} \mathrm{CD} 38^{+} \mathrm{AIM}+\mathrm{CD} 4+\mathrm{T}$ cells was similar to CCC (3.5\% with a $95 \%$ confidence interval of $1.1-7.4 \%)$, with no data point above $10 \%$. These data are consistent with relatively more frequent exposure to influenza as compared to TT responses, and are not consistent with frequenter re-exposure to $\mathrm{CCC}$ of the study cohort, within the time frame of the longitudinal study.

The data above are derived from longitudinal samples collected in the pre-pandemic 20162019 calendar years period. Epidemiological data is available regarding the circulation of CCC in those years, pertaining to the West and Midwest regions ${ }^{12,14}$. If the CCC responses detected were short-lived responses, resulting from frequent re-exposure, we expected that the responses would mirror the CCC circulation pattern when segregated by year. When CCC CD4+ T cell responses were plotted as a function of the year in which the blood donation was obtained, no significant associations using a multiple comparison test, were observed with the yearly incidence of each individual CCC over the same period or when comparing different $\mathrm{CCC}$ responses within a year (Fig. 4C). Overall, these data suggest that CD4+ T CCC-specific responses are not associated with recent activation or frequent yearly reinfections.

\section{CCC circulating antibodies over time confirmed durable antibody titers and infrequent} reinfections

Matched plasma samples were tested for binding of immunoglobulin (Ig) to recombinant Spike RBD antigens from CCC as previously reported ${ }^{44}$. More specifically, we measured the RBD IgG levels by the area under the curve (AUC) in titration experiments for each individual sample

\section{(Fig. S2).}


All subjects were IgG seropositive for the four CCC (NL63, 229E, HKU1 and OC43) at the first and subsequent blood donations, consistent with their high prevalence in human adult populations ${ }^{12,17,45}$ (Fig. 5A). Durability assessments of circulating antibody titers were performed as based curve fits to model ${ }^{41}$, similarly to the assessments of CD4+ T cells responses above. CCC titers were sustained overtime for all the 4 CCC (Fig. 1B); NL63 and 229E titers showed no decline throughout the study, while a modest decline was observed for HKU1 and OC43 with a $\mathrm{t}_{1 / 2}$ of 13.1 and 8.6 years, respectively. The stability of CCC-specific IgG responses indicates that the cohort analyzed was not associated with frequent reinfections during the time considered in the study.

\section{Correlation of CCC-specific CD4+ $\mathrm{T}$ cell and SARS-CoV-2 pre-existing responses}

CCC have significant sequence homology to SARS-CoV-2. The cross-reactive CCCspecific T cell responses with SARS-CoV-2 ${ }^{26,27,46}$ may contribute to modulation of SARS-CoV2 infection and enhance responses to COVID-19 vaccination ${ }^{26,30-33}$. We show that high CD4+ T cell memory OC43 reactivity is associated with higher levels of pre-existing memory reactivity to SARS-CoV-2 (Fig. 6A). Similar patterns were observed for NL63, 229E and HKU1 (Fig. S3) but not for the unrelated and ubiquitous pathogen CMV (Fig. 6B). The highest levels of pre-existing SARS-CoV-2 reactivity were not associated with higher levels of HLA-DR/CD38+ expression (Fig. 6C) suggesting that SARS-CoV-2 cross-reactive cells are not the result of a recent activation or infection. Similarly, high pre-existing SARS-CoV-2 CD4+T cell responses did not correlate with higher IgG antibody reactivity (Fig. 6D). Overall, these results suggest that high CD4+ T cell reactivity to $\mathrm{CCC}$, but not antibody reactivity, is predictive of cross-reactive SARS-CoV-2 CD4+ T cell responses. 


\section{Discussion}

Despite the high prevalence of CCC infection, most adults experience asymptomatic or mild common cold symptoms. Although seroconversion to CCC is near ubiquitous during childhood, little is known about the dynamics of CCC-specific memory responses in adults.

In this study, our goal was to characterize CD4+ T cell-specific memory responses to the four prototypic endemic and widely circulating CCC viruses in a longitudinal cohort, and using as a strategy ex vivo stimulation of PBMCs with peptides pools covering the entire proteome of each individual virus. In particular, our data shows that $\mathrm{T}$ cell reactivity against $\mathrm{CCC}$ is detected for most adult subjects, and that this reactivity is similar in magnitude to other antigens, sustained and relatively constant over time. These observations were paralleled by equally stable circulating Spike RBD antibody titers. While we cannot exclude that at least some reinfections might have occurred during the longitudinal follow up period, the preponderance of evidence suggest that the reactivity observed is associated with memory and persistent responses.

Previous work has suggested that substantial immune memory involving both arms of adaptive immune memory is generated against $\mathrm{CCC}^{17,23,25,26}$. We observed that about $72-81 \%$ of subjects exhibited immune CD4+ T cell memory responses to each of the 4 CCC studied, consistent with frequencies detected in both community and heath care works cohorts ${ }^{26}$, and similar to the findings of Tan et al. ${ }^{25}$. Remarkably, we found a stable and sustained $\mathrm{T}$ cell and antibody response against $\mathrm{CCC}$ which are supported by recent experimental findings from Cohen et al. ${ }^{41}$ or mathematical modeling ${ }^{19,20}$ and argues against the short-lived nature of CCC responses 16-18. Our findings are also in agreement with the stability of responses against other viral infections, such as vaccinia or SARS-CoV-1 where antigen specific cells were detectable 50 years and 17 years post infection, respectively ${ }^{47,48}$ and against TT which are remarkably stable for many years upon vaccination ${ }^{49}$. It is quite possible that the CCC "steady state" reactivity might be result of both repeated infections in early childhood and occasional re-exposure and reinfection.

The data presented herein are also relevant in the context of CCC cross-reactivity with SARS-CoV-2. It has been shown that pre-existing $\mathrm{T}$ cell immunity elicited by past CCC exposures can influence COVID-19 responsiveness to vaccination and disease outcome ${ }^{30-33,35}$. We found that while everybody had detectable antibody titers to CCC individuals varied in the level of T cell reactivity (possibly as a function of recent exposure, HLA type or other individual and environmental factors), and that the subjects with high CCC T cell reactivity, but not antibody 
titers, are those most likely to be associated with pre-existing SARS-CoV-2 immune reactivity. This is consistent with findings that CCC antibodies might not protect against SARS-CoV-2 infection or disease severity ${ }^{24,50}$ but $\mathrm{T}$ cells do ${ }^{30,51}$.

SARS-CoV-2 antibody and T cell effector activity contract over time and protection from infection wanes but protection from severe disease appears to be preserved in significant degree ${ }^{8,52-54}$. Likewise, it has also been suggested that recurrent CCC infections are only rarely associated with moderate or severe clinical symptoms ${ }^{17,21}$. In the context of the debate of the evolution of SARS-CoV-2 pandemic, our data are compatible with the notion upon enough people being exposed and vaccinated, SARS-CoV-2 at some point might turn endemic and resemble CCC, in terms of sustained herd immunity and relative protection not from infection but from symptomatic and severe disease. The development of pan-corona vaccines that target not only SARS-CoV-2 but also CCC viruses might contribute to further protection.

In summary, we found that in addition to widespread antibody reactivity to all the four $\mathrm{CCC}$, memory $\mathrm{T}$-cell responses are detected for most individuals and their reactivity remained stable and relatively constant over time. The characterization of the immune response to the prevalent and endemic CCCs provides a valuable reference for understanding the durability and eventual transition to an endemic state of SARS-CoV-2 in the aftermath of the pandemic.

\section{Limitations of the study}

A limitation of this investigation is the unknown history of previous CCC exposure of the study participants. Assessment of CCC infection by RT-PCR was not part of the original study design for the study, and would also not have been logistically feasible over the longitudinal course of the study as it would have required frequent nasal swabs of all subjects. Therefore, in the present study, the stability of $\mathrm{T}$ cell and antibody responses could not be directly correlated with protection from symptomatic colds and/or infection. Furthermore, our analysis is limited to "steady state" responses in adults, and the evolution of CCC responses in children was not addressed. Additional limitations of this study are the relatively small cohort size investigated. Validation of the results in geographically distinct populations would be desirable to generalize the findings broadly. 


\section{Acknowledgments}

We wish to acknowledge all subjects for their participation and for donating their blood and time for this study. We are grateful to the La Jolla Institute for Immunology clinical core relentless efforts in obtaining blood samples. Research reported in this publication was supported by the National Institute of Allergy and Infectious Diseases (NIAID) of the National Institutes of Health (NIH) under Award numbers U01AI141995, U19AI142742 and U54CA260543, and contract number 75N93019C00065. The content is solely the responsibility of the authors and does not necessarily represent the official views of the National Institutes of Health.

\section{Author Contributions}

Designing research studies, AS, RdSA, EDY; Investigation, AS, RdSA, EDY, TN, EW, EG, LP, JM; Data Analysis, EDY, RdSA, LP; Resources, AS and RdSA; Manuscript writing, AS, RdSA, EDY, LP, DW, AG; Supervision, AS and RdSA; Project Administration, AF; Funding Acquisition, AS and RdSA.

\section{Declaration of Interests}

A.Se. is a consultant for Gritstone Bio, Flow Pharma, Arcturus Therapeutics, ImmunoScape, CellCarta, Avalia, Moderna, Fortress and Repertoire. S.C. is a consultant for Avalia. LJI has filed for patent protection for various aspects of SARS-CoV-2 epitope pools design. All other authors declare no conflict of interest. 


\section{Materials and methods}

\section{Study cohort and PBMC isolation}

The purpose of this study was to investigate the immunological memory to common cold corona viruses in a longitudinal cohort collected during pre-pandemic time. Blood donations from 32 donors previously recruited in a Bordetella Pertussis observational study were collected under IRB approved protocols at the La Jolla Institute for Immunology (protocol no. VD-101), before COVID-19 pandemic from 2016-2019. There were 3 to 7 longitudinal blood donations per donor spanning time periods from 6 months to more than 3 years. Each participant provided informed consent and was assigned a study identification number with clinical information recorded. Exclusion criteria included pregnancy at the start of the study; presentation of severe disease; medical treatment that might interfere with study results and/or antibiotic use or fever $\left(>100.4^{\circ} \mathrm{F}\right.$ $\left.\left[38^{\circ} \mathrm{C}\right]\right)$. No exclusions were made due to race, ethnicity, or gender. In all cases, PBMCs were isolated from whole blood by density gradient centrifugation according to manufacturer instructions (Ficoll-Hypaque, Amersham Biosciences, Uppsala, Sweden) and cryopreserved for further analysis.

\section{Synthesize of epitope pools}

In the current study, in order to investigate the CCC and SARS-CoV-2 specific T cell responses, we used megapools (MPs) combing the overlapping spike (S) epitope pools and predicted HLA class II CD4+ T cell epitope pools from the rest of the genome (R) (Table 2), generated using previously described strategies ${ }^{26,36}$. We also studied antigen-specific responses against a panel of other respiratory viruses (influenza, RSV, and rhinovirus), chronically infectious viruses (EBV, CMV, and VZV), and ubiquitous bacterial vaccine antigens (TT and PT) using peptide sets described in Table 2. Detailed overall information of the MPs composition, peptide numbers as well as references are specified in Table 2. Individual peptides were synthesized by TC peptide lab (San Diego, CA) and pooled by protein combinations and resuspended to a final concentration of $1 \mathrm{mg} / \mathrm{mL}$ in DMSO.

\section{Spike protein RBD Enzyme-Linked Immunosorbent Assay}

All plasma samples tested by ELISA assay were heat-inactivated at $56^{\circ} \mathrm{C}$ for $30 \mathrm{~min}$ to reduce risk from any possible residual virus in serum. Briefly, $50 \mu \mathrm{L}$ of Streptavidin (Invitrogen) 
at $4 \mu \mathrm{g} / \mathrm{mL}$ in Tris-Buffered Saline (TBS) $\mathrm{pH} 7.4$ was coated in the 96-well, high-binding microtiter assay plate (Greiner Bio-One cat \# 655061) for 1 hour at $37^{\circ} \mathrm{C}$. The coating solution was removed, then $100 \mu \mathrm{L}$ of blocking solution, 1:1 Non Animal Protein-BLOCKER ${ }^{\mathrm{TM}}$ (GBiosciences) in TBS was added for 1 hour at $37^{\circ} \mathrm{C}$. Serum samples were serially diluted (1:40 1:8100), in 3\% Bovine Serum Albumin (BSA) in TBS containing 0.05\% Tween 20 (TBST) with respective biotinylated spike $\mathrm{RBD}$ antigens from $\mathrm{CCC}$ at $1 \mu \mathrm{g} / \mathrm{mL}$ in a 96 -round-well $\mathrm{V}$ bottom plate (Diaago cat \# R96-300V) and incubated for 1 hour at $37^{\circ} \mathrm{C}$. The blocking solution was removed, then $50 \mu \mathrm{L}$ of diluted serum was added to the assay plate and incubated for 15 minutes at $37^{\circ} \mathrm{C}$. The plate was washed three times using wash buffer (1X TBS containing $0.2 \%$ Tween 20), then $50 \mu \mathrm{L}$ of horseradish peroxidase-conjugated secondary Goat Anti-Human secondary IgG antibody (Cat No: 109-035-008, Jackson ImmunoResearch) at 1:40,000 dilution in 3\% milk was added for 1 hour at $37^{\circ} \mathrm{C}$. The plate was washed three times using wash buffer, then $50 \mu \mathrm{L}$ of 3,3',5,5' -Tetramethylbenzidine (TMB) Liquid Substrate (Sigma-Aldrich) was added to the plate, and absorbance was measured at $450 \mathrm{~nm}$ using a plate reader (Molecular Devices SpectraMax ABS Plus Absorbance ELISA Mcroplate Reader) after stopping the reaction with $50 \mu$ of $1 \mathrm{~N}$ $\mathrm{HCl}$. Area under the curve for titration experiments for each sample were calculated by the trapezoidal model implemented in Prism Version 9.3.0.

\section{Activation induced cell marker (AIM) assay}

The AIM assay was performed as previously described ${ }^{27}$. Cryopreserved PBMCs were thawed by diluting the cells in $10 \mathrm{~mL}$ complete RPMI 1640 with 5\% human AB serum (Gemini Bioproducts) in the presence of benzonase [20 ml/10ml]. Cells were cultured for 20 to 24 hours in the presence of CCC or SARS-CoV-2 specific and other common antigen pools $(1 \mathrm{ug} / \mathrm{ml})$ in 96wells U bottom plates with $1 \times 10^{6}$ PBMC per well. An equimolar amount of DMSO was added as a negative control and phytohemagglutinin (PHA, Roche (San Diego, CA) $1 \mathrm{mg} / \mathrm{ml}$ ) was used as the positive control. Cells were stained and activation of CD4+ T cell measured by the CD137 and OX40 marker combination. The detailed information of all the antibodies used are summarized in Table S1. All samples were acquired on a ZE5 cell analyzer (Biorad laboratories, Hercules, CA) and analyzed with FlowJo software (Tree Star, Ashland, OR). AIM+ CD4+ T cells data were calculated as percent of total CD4+ $\mathrm{T}$ cells background subtracted or stimulation index. Background subtracted data were derived by subtracting the percentage of AIM + cells percentage 
after each MP stimulation from the DMSO stimulation. The Stimulation Index (SI) was calculated by dividing the count of AIM+ cells after SARS-CoV-2 pools stimulation with the ones in the negative control. A positive response was defined as SI greater than 2 and AIM+ response above the threshold of positivity after background subtraction. The limit of detection $(0.02 \%)$ was calculated based on 2 times $95 \%$ CI of geomean of negative control (DMSO), and the threshold of positivity $(0.03 \%)$ was calculated based on 2 times standard deviation of background signals according to previous published studies ${ }^{27,52}$. All data below 0.02 or $\mathrm{SI}<2$ were set to 0.02 or 2 for plotting and statistical analysis. The detailed gating strategy used to define CD4+ AIM reactive cells $\left(\mathrm{OX} 40^{+} \mathrm{CD} 137^{+}\right)$, memory (CD45RA/CCR7), and activated sub-populations (HLA$\mathrm{DR}+\mathrm{CD} 38+$ ) is listed in Fig. $\mathbf{S 4}$. Gates were drawn relative to the unstimulated condition for each donor.

\section{Statistical analysis}

Experimental data were analyzed by GraphPad Prism Version 9 (La Jolla, CA) and Microsoft Excel Version 16.16.27 (Microsoft, Redmond, WA). The statistical details of the experiments are provided in the respective figure legends. Data were analyzed by Mann-Whitney test (two-tailed) to compare between two groups, and Kruskal-Wallis test adjusted with Dunn's test for multiple comparisons to compare between multiple groups. The regression lines and estimated $t 1 / 2$ was calculated based on linear mixed effects model using $\mathrm{R}$ package nlme as previously described by Cohen et al. $2021^{41}$. Data were plotted as geometric mean with geometric SD for log scale and median with interquartile range for numeric scale. $p$ values $<0.05$ (after adjustment if indicated) were considered statistically significant.

\section{Study approval}

This study was approved under IRB protocol approval (VD-101) at the La Jolla Institute for Immunology. All donors were able to provide informed consent, or had a legal guardian or representative able to do so. Each participant provided informed consent and was assigned a study identification number with clinical information recorded. 


\section{Materials \& Correspondence}

Epitope pools used in this study will be made available to the scientific community upon request, and following execution of a material transfer agreement (MTA), by contacting A.S. (alex@1ji.org) and R.d.S.A (rantunes@lji.org). Likewise, biomaterials archived from this study may be shared for further research with MTA.

\section{Data and code availability}

The datasets generated and analyzed in this study will be shared by the corresponding authors upon reasonable request. This paper does not report original code. 


\section{References}

1 van der Hoek, L. et al. Identification of a new human coronavirus. Nat Med 10, 368-373, doi:10.1038/nm1024 (2004).

2 Walsh, E. E., Shin, J. H. \& Falsey, A. R. Clinical impact of human coronaviruses 229E and OC43 infection in diverse adult populations. J Infect Dis 208, 1634-1642, doi:10.1093/infdis/jit393 (2013).

3 Woo, P. C. et al. Characterization and complete genome sequence of a novel coronavirus, coronavirus HKU1, from patients with pneumonia. J Virol 79, 884-895, doi:10.1128/JVI.79.2.884-895.2005 (2005).

4 Graat, J. M. et al. A prospective, community-based study on virologic assessment among elderly people with and without symptoms of acute respiratory infection. J Clin Epidemiol 56, 1218-1223, doi:10.1016/s0895-4356(03)00171-9 (2003).

5 Pene, F. et al. Coronavirus 229E-related pneumonia in immunocompromised patients. Clin Infect Dis 37, 929-932, doi:10.1086/377612 (2003).

6 Cui, J., Li, F. \& Shi, Z. L. Origin and evolution of pathogenic coronaviruses. Nat Rev Microbiol 17, 181-192, doi:10.1038/s41579-018-0118-9 (2019).

7 Galanti, M. et al. Longitudinal active sampling for respiratory viral infections across age groups. Influenza Other Respir Viruses 13, 226-232, doi:10.1111/irv.12629 (2019).

8 Siggins, M. K., Thwaites, R. S. \& Openshaw, P. J. M. Durability of Immunity to SARSCoV-2 and Other Respiratory Viruses. Trends Microbiol 29, 648-662, doi:10.1016/j.tim.2021.03.016 (2021).

9 Wang, Y., Grunewald, M. \& Perlman, S. Coronaviruses: An Updated Overview of Their Replication and Pathogenesis. Methods Mol Biol 2203, 1-29, doi:10.1007/978-1-07160900-2_1 (2020).

10 Dominguez, S. R., Robinson, C. C. \& Holmes, K. V. Detection of four human coronaviruses in respiratory infections in children: a one-year study in Colorado. $J$ Med Virol 81, 1597-1604, doi:10.1002/jmv.21541 (2009).

11 Selva, K. J. et al. Systems serology detects functionally distinct coronavirus antibody features in children and elderly. Nat Commun 12, 2037, doi:10.1038/s41467-021-22236-7 (2021).

12 Killerby, M. E. et al. Human coronavirus circulation in the United States 2014-2017. J Clin Virol 101, 52-56, doi:10.1016/j.jcv.2018.01.019 (2018).

13 Li, Y., Wang, X. \& Nair, H. Global Seasonality of Human Seasonal Coronaviruses: A Clue for Postpandemic Circulating Season of Severe Acute Respiratory Syndrome Coronavirus 2? J Infect Dis 222, 1090-1097, doi:10.1093/infdis/jiaa436 (2020).

14 Rucinski, S. L., Binnicker, M. J., Thomas, A. S. \& Patel, R. Seasonality of Coronavirus 229E, HKU1, NL63, and OC43 From 2014 to 2020. Mayo Clin Proc 95, 1701-1703, doi:10.1016/j.mayocp.2020.05.032 (2020).

15 Park, S., Lee, Y., Michelow, I. C. \& Choe, Y. J. Global Seasonality of Human Coronaviruses: A Systematic Review. Open Forum Infect Dis 7, ofaa443, doi:10.1093/ofid/ofaa443 (2020).

16 Callow, K. A., Parry, H. F., Sergeant, M. \& Tyrrell, D. A. The time course of the immune response to experimental coronavirus infection of man. Epidemiol Infect 105, 435-446, doi:10.1017/s0950268800048019 (1990). 
17 Edridge, A. W. D. et al. Seasonal coronavirus protective immunity is short-lasting. Nat Med 26, 1691-1693, doi:10.1038/s41591-020-1083-1 (2020).

18 Kissler, S. M., Tedijanto, C., Goldstein, E., Grad, Y. H. \& Lipsitch, M. Projecting the transmission dynamics of SARS-CoV-2 through the postpandemic period. Science 368, 860-868, doi:10.1126/science.abb5793 (2020).

19 Townsend, J. P. et al. The durability of immunity against reinfection by SARS-CoV-2: a comparative evolutionary study. Lancet Microbe 2, e666-e675, doi:10.1016/S26665247(21)00219-6 (2021).

20 Waterlow, N. R. et al. How immunity from and interaction with seasonal coronaviruses can shape SARS-CoV-2 epidemiology. Proc Natl Acad Sci U S A 118, doi:10.1073/pnas.2108395118 (2021).

21 Galanti, M. \& Shaman, J. Direct Observation of Repeated Infections With Endemic Coronaviruses. J Infect Dis 223, 409-415, doi:10.1093/infdis/jiaa392 (2021).

22 Petrie, J. G. et al. Coronavirus Occurrence in the Household Influenza Vaccine Evaluation (HIVE) Cohort of Michigan Households: Reinfection Frequency and Serologic Responses to Seasonal and Severe Acute Respiratory Syndrome Coronaviruses. J Infect Dis 224, 49-59, doi:10.1093/infdis/jiab161 (2021).

23 Khan, T. et al. Distinct antibody repertoires against endemic human coronaviruses in children and adults. JCI Insight 6, doi:10.1172/jci.insight.144499 (2021).

24 Lin, C. Y. et al. Pre-existing humoral immunity to human common cold coronaviruses negatively impacts the protective SARS-CoV-2 antibody response. Cell Host Microbe 30, 83-96 e84, doi:10.1016/j.chom.2021.12.005 (2022).

25 Tan, H. X. et al. Adaptive immunity to human coronaviruses is widespread but low in magnitude. Clin Transl Immunology 10, e1264, doi:10.1002/cti2.1264 (2021).

26 da Silva Antunes, R. et al. Differential T-Cell Reactivity to Endemic Coronaviruses and SARS-CoV-2 in Community and Health Care Workers. J Infect Dis 224, 70-80, doi:10.1093/infdis/jiab176 (2021).

27 Mateus, J. et al. Selective and cross-reactive SARS-CoV-2 T cell epitopes in unexposed humans. Science 370, 89-94, doi:10.1126/science.abd3871 (2020).

28 Dykema, A. G. et al. Functional characterization of CD4+ T cell receptors crossreactive for SARS-CoV-2 and endemic coronaviruses. J Clin Invest 131, doi:10.1172/JCI146922 (2021).

29 Woldemeskel, B. A. et al. CD4+ T-cells from COVID-19 mRNA vaccine recipients recognize a conserved epitope present in diverse coronaviruses. $J$ Clin Invest, doi:10.1172/JCI156083 (2022).

30 Kundu, R. et al. Cross-reactive memory T cells associate with protection against SARSCoV-2 infection in COVID-19 contacts. Nat Commun 13, 80, doi:10.1038/s41467-02127674-x (2022).

31 Loyal, L. et al. Cross-reactive CD4(+) T cells enhance SARS-CoV-2 immune responses upon infection and vaccination. Science 374, eabh1823, doi:10.1126/science.abh1823 (2021).

32 Mateus, J. et al. Low-dose mRNA-1273 COVID-19 vaccine generates durable memory enhanced by cross-reactive T cells. Science 374, eabj9853, doi:10.1126/science.abj9853 (2021).

33 Sagar, M. et al. Recent endemic coronavirus infection is associated with less-severe COVID-19. J Clin Invest 131, doi:10.1172/JCI143380 (2021). 
$34 \mathrm{Su}, \mathrm{S} ., \mathrm{Li}, \mathrm{W}$. \& Jiang, S. Developing pan-beta-coronavirus vaccines against emerging SARS-CoV-2 variants of concern. Trends Immunol, doi:10.1016/j.it.2022.01.009 (2022).

35 da Silva Antunes, R. et al. Th1/Th17 polarization persists following whole-cell pertussis vaccination despite repeated acellular boosters. J Clin Invest 128, 3853-3865, doi:10.1172/JCI121309 (2018).

36 Grifoni, A. et al. Targets of T Cell Responses to SARS-CoV-2 Coronavirus in Humans with COVID-19 Disease and Unexposed Individuals. Cell 181, 1489-1501 e1415, doi:10.1016/j.cell.2020.05.015 (2020).

37 Dhanwani, R. et al. Profiling Human Cytomegalovirus-Specific T Cell Responses Reveals Novel Immunogenic Open Reading Frames. J Virol 95, e0094021, doi:10.1128/JVI.00940-21 (2021).

38 Tarke, A. et al. Comprehensive analysis of T cell immunodominance and immunoprevalence of SARS-CoV-2 epitopes in COVID-19 cases. Cell Rep Med 2, 100204, doi:10.1016/j.xcrm.2021.100204 (2021).

39 Voic, H. et al. Identification and Characterization of CD4(+) T Cell Epitopes after Shingrix Vaccination. J Virol 94, doi:10.1128/JVI.01641-20 (2020).

$40 \mathrm{Yu}, \mathrm{E}$. D. et al. Development of a T cell-based immunodiagnostic system to effectively distinguish SARS-CoV-2 infection and COVID-19 vaccination status. Cell Host Microbe, doi:10.1016/j.chom.2022.02.003 (2022).

41 Cohen, K. W. et al. Longitudinal analysis shows durable and broad immune memory after SARS-CoV-2 infection with persisting antibody responses and memory B and T cells. Cell Rep Med 2, 100354, doi:10.1016/j.xcrm.2021.100354 (2021).

42 Kuri-Cervantes, L. et al. Comprehensive mapping of immune perturbations associated with severe COVID-19. Sci Immunol 5, doi:10.1126/sciimmunol.abd7114 (2020).

43 Sekine, T. et al. Robust T Cell Immunity in Convalescent Individuals with Asymptomatic or Mild COVID-19. Cell 183, 158-168 e114, doi:10.1016/j.cell.2020.08.017 (2020).

44 Premkumar, L. et al. The receptor binding domain of the viral spike protein is an immunodominant and highly specific target of antibodies in SARS-CoV-2 patients. Sci Immunol 5, doi:10.1126/sciimmunol.abc8413 (2020).

45 Nickbakhsh, S. et al. Epidemiology of Seasonal Coronaviruses: Establishing the Context for the Emergence of Coronavirus Disease 2019. J Infect Dis 222, 17-25, doi:10.1093/infdis/jiaa185 (2020).

46 Low, J. S. et al. Clonal analysis of immunodominance and cross-reactivity of the CD4 T cell response to SARS-CoV-2. Science 372, 1336-1341, doi:10.1126/science.abg8985 (2021).

47 Demkowicz, W. E., Jr., Littaua, R. A., Wang, J. \& Ennis, F. A. Human cytotoxic T-cell memory: long-lived responses to vaccinia virus. J Virol 70, 2627-2631, doi:10.1128/JVI.70.4.2627-2631.1996 (1996).

48 Le Bert, N. et al. SARS-CoV-2-specific T cell immunity in cases of COVID-19 and SARS, and uninfected controls. Nature 584, 457-462, doi:10.1038/s41586-020-2550-z (2020).

49 Hammarlund, E. et al. Durability of Vaccine-Induced Immunity Against Tetanus and Diphtheria Toxins: A Cross-sectional Analysis. Clin Infect Dis 62, 1111-1118, doi:10.1093/cid/ciw066 (2016). 
50 Wratil, P. R. et al. Evidence for increased SARS-CoV-2 susceptibility and COVID-19 severity related to pre-existing immunity to seasonal coronaviruses. Cell Rep 37, 110169, doi:10.1016/j.celrep.2021.110169 (2021).

51 Mallajosyula, V. et al. CD8(+) T cells specific for conserved coronavirus epitopes correlate with milder disease in COVID-19 patients. Sci Immunol 6, doi:10.1126/sciimmunol.abg5669 (2021).

52 Dan, J. M. et al. Immunological memory to SARS-CoV-2 assessed for up to 8 months after infection. Science 371, doi:10.1126/science.abf4063 (2021).

53 Ortega, N. et al. Seven-month kinetics of SARS-CoV-2 antibodies and role of preexisting antibodies to human coronaviruses. Nat Commun 12, 4740, doi:10.1038/s41467021-24979-9 (2021).

54 Sette, A. \& Crotty, S. Adaptive immunity to SARS-CoV-2 and COVID-19. Cell 184, 861-880, doi:10.1016/j.cell.2021.01.007 (2021).

55 Dhanda, S. K. et al. IEDB-AR: immune epitope database-analysis resource in 2019. Nucleic Acids Res 47, W502-W506, doi:10.1093/nar/gkz452 (2019).

56 Grifoni, A. et al. A survey of known immune epitopes in the enteroviruses strains associated with acute flaccid myelitis. Hum Immunol 80, 923-929, doi:10.1016/j.humimm.2019.08.004 (2019).

57 da Silva Antunes, R. et al. Definition of Human Epitopes Recognized in Tetanus Toxoid and Development of an Assay Strategy to Detect Ex Vivo Tetanus CD4+ T Cell Responses. PLoS One 12, e0169086, doi:10.1371/journal.pone.0169086 (2017).

58 da Silva Antunes, R. et al. Development and Validation of a Bordetella pertussis WholeGenome Screening Strategy. J Immunol Res 2020, 8202067, doi:10.1155/2020/8202067 (2020). 


\section{Figures and Tables}

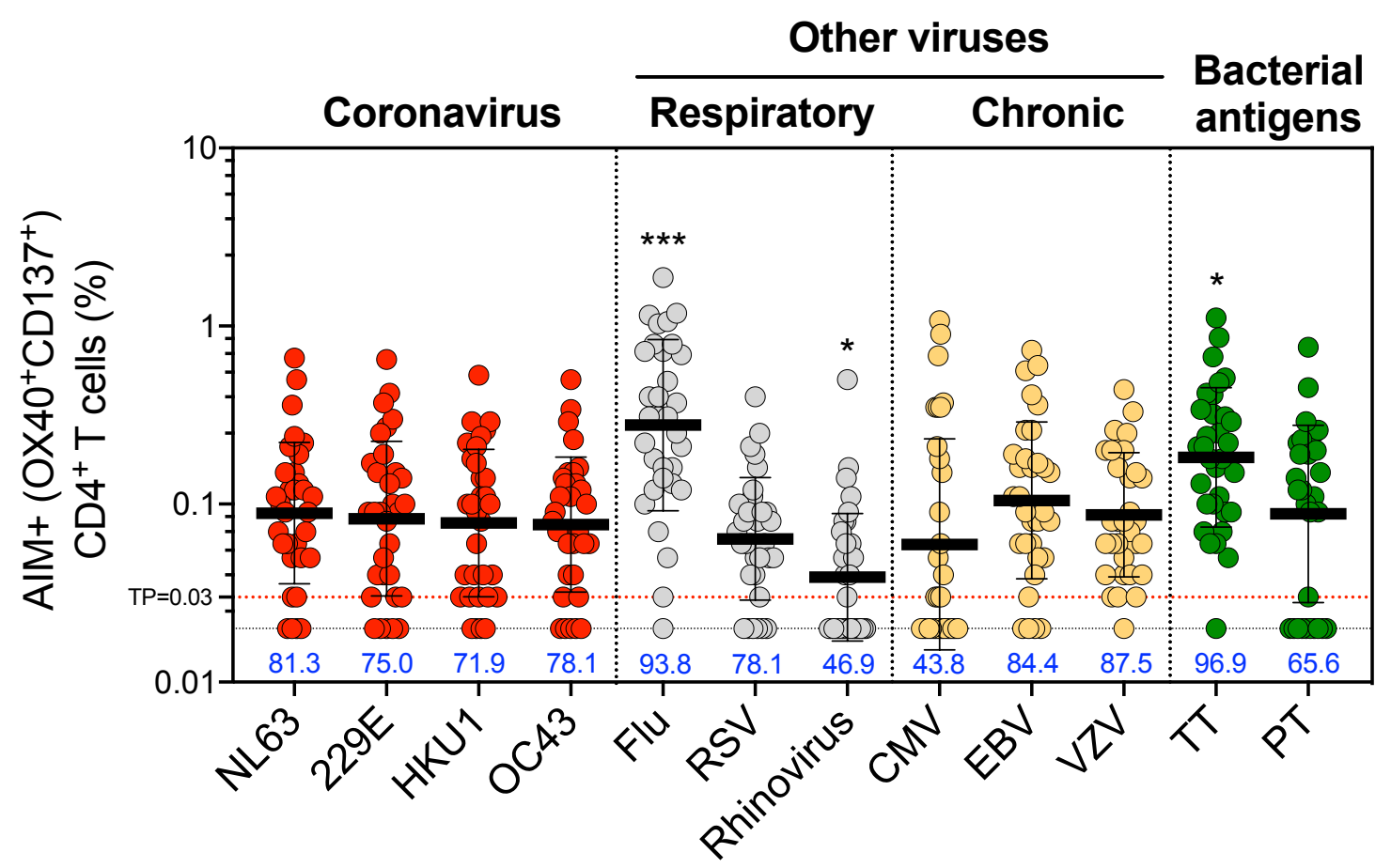

Fig. $1 \mathrm{CD} 4+\mathrm{T}$ cell responses to four representative $\mathrm{CCC}$ are widely detectable in the study cohort and of similar magnitude to other pathogens. Common cold coronavirus (CCC) and several other human pathogens-specific T cell responses were measured as percentage of AIM+ $(\mathrm{OX} 40+\mathrm{CD} 137+)$ CD4+ T cells after stimulation of PBMCs with peptides pools. Graphs show individual response of the four CCC (NL63, 229E, HKU1, and OC43) and other pathogens plotted as background subtracted against DMSO negative control. First time point of the longitudinal series is plotted $(n=32)$ and associated percentage of positive response for each antigen is indicated. $\mathrm{TP}=$ threshold of positivity. Data are represented as geometric mean and SD. KruskalWallis test adjusted with Dunn's test for multiple comparisons was performed and adjusted $\mathrm{p}$ values $<0.05$ considered statistically significant. ${ }^{*}, \mathrm{p}<0.05, * * *, \mathrm{p}<0.001$. 
A
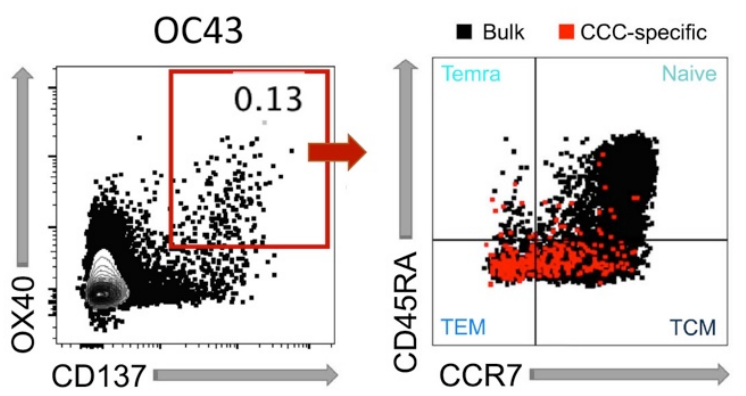

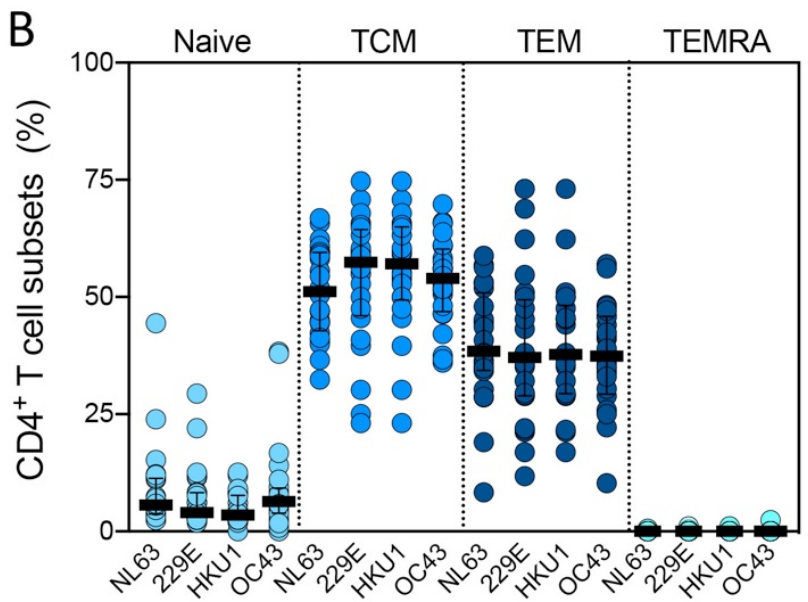

Fig. 2 CCC-specific CD4+ T cells are largely classic memory CD4 T cells. CCC-specific CD4+ T cell subsets (Tn: CD45RA+ CCR7+, Temra: CD45RA+ CCR7-, Tcm: CD45RA- CCR7+ and Tem: CD45RA- CCR7-) were measured after stimulation of PBMCs with specific peptide pools. (A) Representative FACS plots, gated on the CCC-specific CD4+ T cells (red) measured as percentage of AIM+ $(\mathrm{OX} 40+\mathrm{CD} 137+)$ from total CD4 $\mathrm{T}$ cells (Left), with the four subsets indicated in each quadrant for AIM+ cells (red) or total CD4+ T cells (black) (Right) are shown. (B) Percentages of T cell subsets from antigen-specific CD4+ T cells (OX40+CD137+) responding to the indicated pools of CCC and with SI $>2$ in each cohort $(n=32)$ at first time point are shown. Each dot represents the response of an individual subject to an individual pool and error bars represent median with interquantile range. 

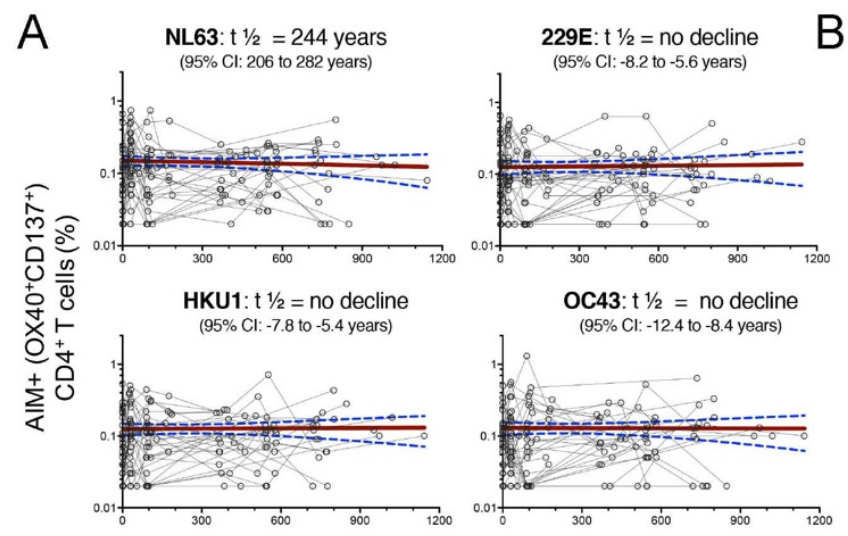

B

C

Days since follow up
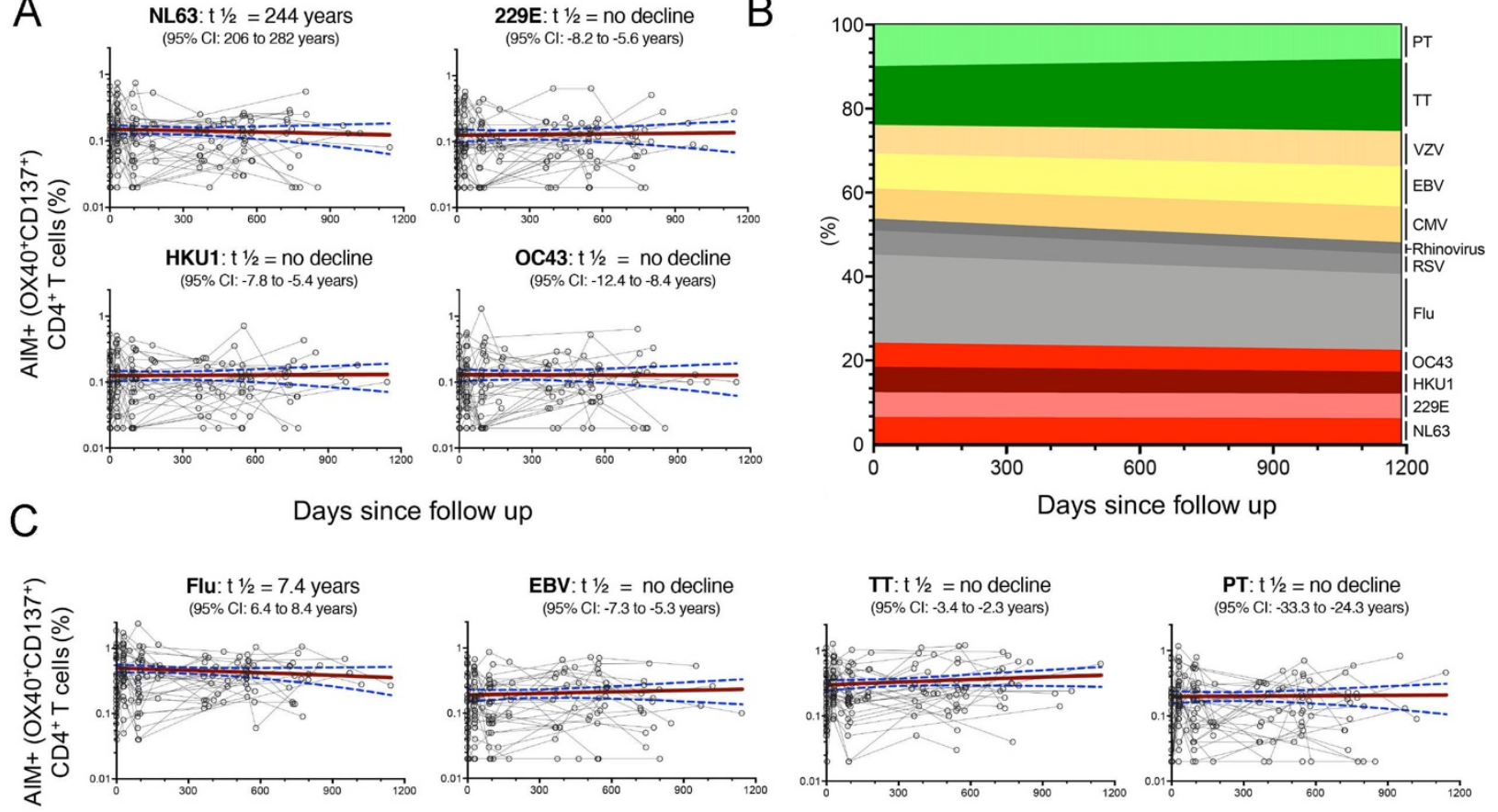

Days since follow up

Fig. 3 CD4+ T cells responses to $\mathrm{CCC}$ and other antigens are sustained over time. Antigenspecific $\mathrm{T}$ cell responses were measured as percentage of AIM+ (OX40+CD137+) CD4+ T cells after stimulation of PBMCs with peptides pools. Individual responses of the four CCC $(\mathbf{A}, \mathbf{B})$ or other pathogens $(\mathbf{B}, \mathbf{C})$ are shown. $(\mathbf{A}, \mathbf{C})$ Graphs show responses plotted with all time points of the longitudinal series connected with lines for each subject $(n=32)$. The red line represents the median fitted curve from a nonlinear mixed effects model of longitudinal responses among those with a positive response at $\geq 1$ time point, with $95 \% \mathrm{CI}$ shown in blue dotted lines. $\mathrm{t} 1 / 2$ calculated based on linear mixed effects model using $\mathrm{R}$ package nlme ${ }^{41}, \mathrm{t}^{1 / 2}$ is shown as the median half-life estimated from the median slope with the associated 95\% CI indicated. (B) Longitudinal occurrence of each individual pathogen response distributed in overall percentage (sum of all absolute responses) in relation to the days since follow up. 
A

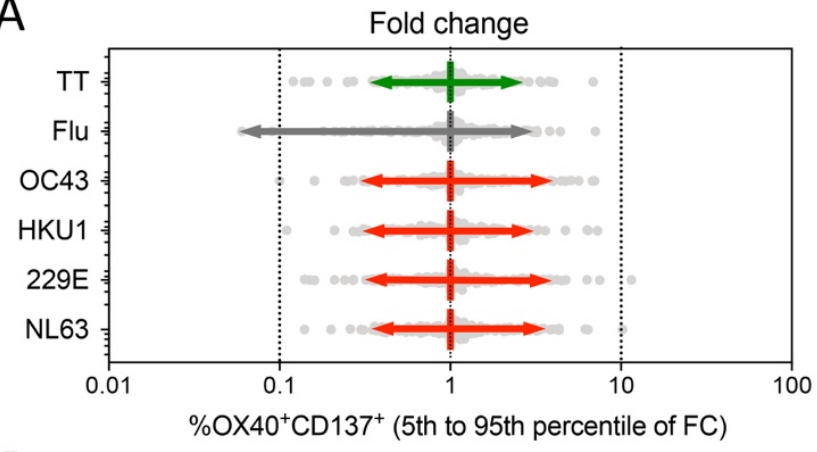

B

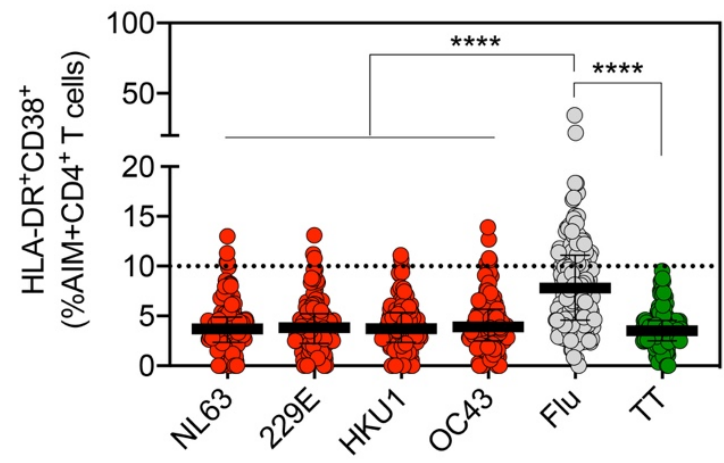

C

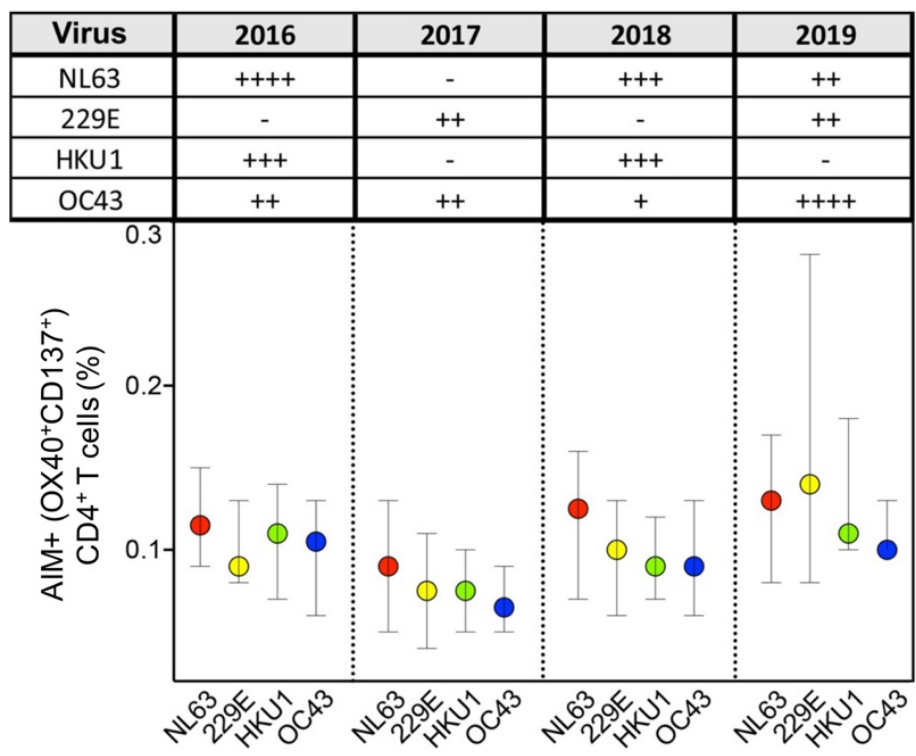

Fig. 4 CCC-specific CD4+ $T$ cell responses are stable, not associated with recent activation or yearly changes in prevalence of CCC infections. (A) The range of fluctuation of CD4+ T cell responses was determined by calculating the fold change of antigen-specific AIM+ $(\mathrm{OX} 40+\mathrm{CD} 137+) \mathrm{CD} 4+\mathrm{T}$ cells. For each antigen, AIM+CD4+ responses at every time point were normalized to median of total longitudinal responses for each donor $(\mathrm{n}=32)$, and the $5^{\text {th }}$ to $95^{\text {th }}$ percentile range calculated. (B) Graph shows CCC, influenza and tetanus specific CD4+ T cell responses associated with recent activation measured by calculating the $\%$ of $\mathrm{HLA}_{-\mathrm{DR}}^{+} \mathrm{CD} 38^{+}$ from AIM $+\left(\mathrm{OX} 40^{+} \mathrm{CD} 137^{+}\right) \mathrm{CD} 4+\mathrm{T}$ cells at all time points of the longitudinal cohort. Each dot represents the response of an individual subject $(n=32)$ to an individual pool at a single time point. Median and interquartile range are represented. (C) The prevalences of CCC infections in the west and midwest regions during 2016-2019 were categorized according to the percent of positive rates from total tests performed ${ }^{12,14}:-,<1 \% ;+, 1-2 \%,++, 2-5 \% ;+++, 5-8 \% ;++++,>8 \%$, and results summarized in the table insert. CCC-specific CD4+ T cell responses for the four CCC were ploted as function of the yearly incidence (2016-2019) in the graph below. Median and interquartile range are represented. Kruskal-Wallis test adjusted with Dunn's test for multiple comparisons was performed and adjusted $\mathrm{p}$ values $<0.05$ considered statistically significant. 

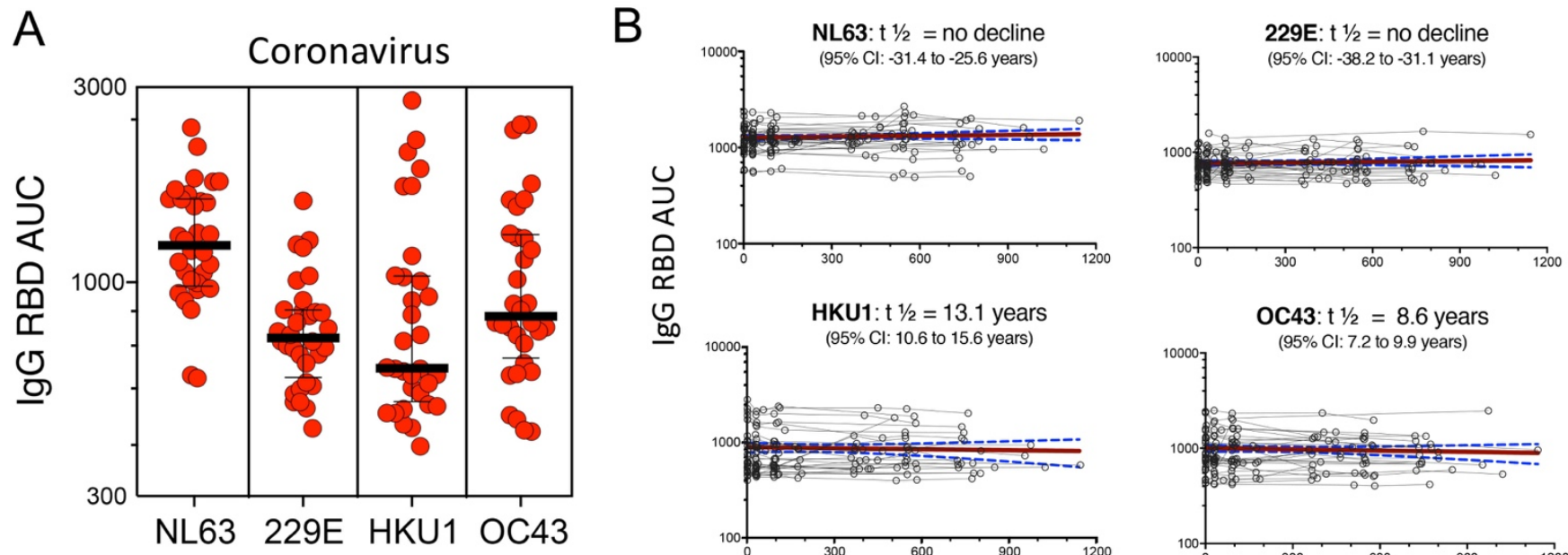

HKU1: $\mathrm{t} \mathrm{t} 1 / 2=13.1$ years
(95\% $\mathrm{Cl}: 10.6$ to 15.6 years $)$
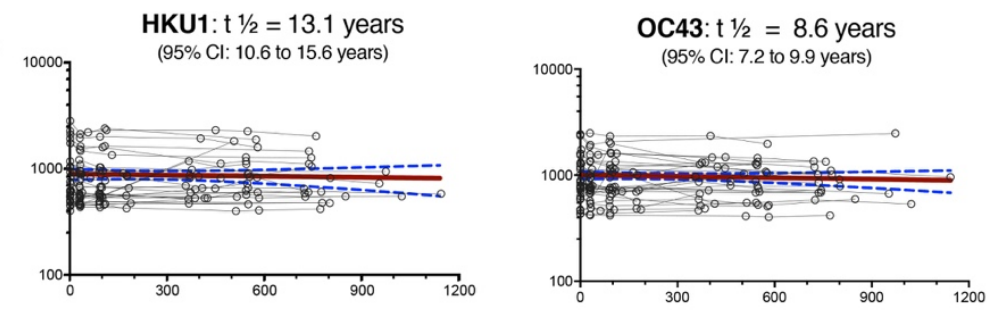

Fig. 5 CCC-specific IgG responses are detected in all individuals and sustained overtime. (A)

Plasma IgG titers, measured by the AUC, to the spike receptor binding domain (RBD) protein of the $\mathrm{CCC}$ viruses (HCoV-229E, HCoV-NL63, HCoV-HKU1 and $\mathrm{HCoV}-\mathrm{OC} 43)$ are shown for first time point of the longitudinal cohort $(n=32)$. Geometric mean titers with SD are indicated. (B) Graphs show individual CCC antibody responses plotted for all time points of the longitudinal series and connected with lines for each subject $(n=32)$. The red line represents the median fitted curve from a nonlinear mixed effects model of longitudinal responses among those with a positive response at $\geq 1$ time point, with $95 \%$ CI shown in blue dotted lines. $t \frac{1}{2}$ calculated based on linear mixed effects model using $\mathrm{R}$ package nlme ${ }^{41}, \mathrm{t}^{1 / 2}$ is shown as the median half-life estimated from the median slope with the associated $95 \% \mathrm{CI}$ indicated. 


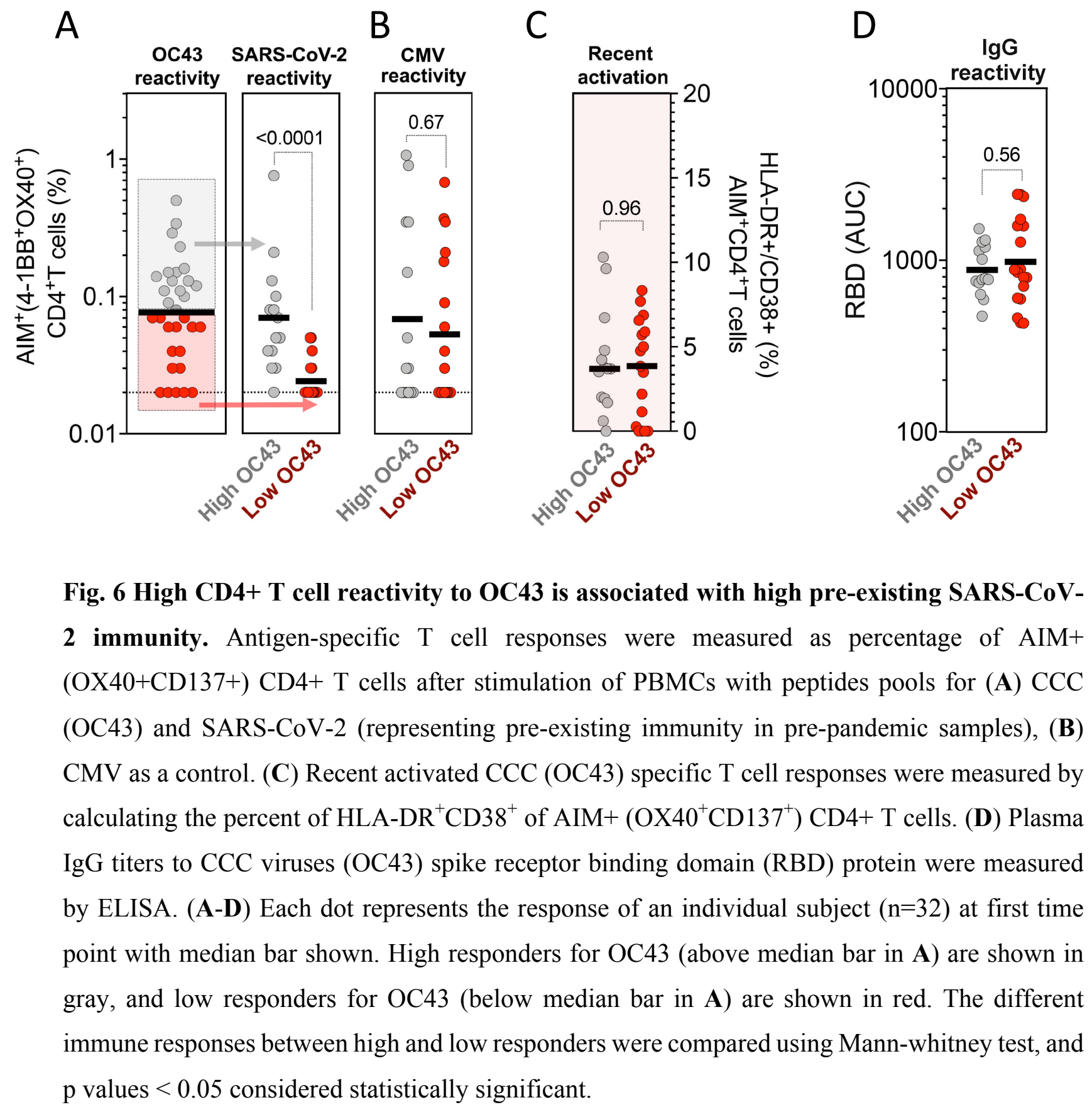


bioRxiv preprint doi: https://doi.org/10.1101/2022.03.01.482548; this version posted March 2, 2022. The copyright holder for this preprint (which was not certified by peer review) is the author/funder, who has granted bioRxiv a license to display the preprint in perpetuity. It is made available under aCC-BY-NC-ND 4.0 International license.

Table 1. Overall characteristics of the study cohort

\begin{tabular}{|c|c|c|c|c|c|c|c|}
\hline $\begin{array}{c}\text { Number of } \\
\text { Donors }\end{array}$ & $\begin{array}{c}\text { Age } \\
\text { median and } \\
\text { range) }\end{array}$ & \multicolumn{2}{|c|}{ Gender } & \multicolumn{3}{|c|}{ Ethnicity } \\
\hline & & Male & Female & Caucasian & $\begin{array}{c}\text { Hispanic / } \\
\text { Latino }\end{array}$ & Asian & $\begin{array}{c}\text { African } \\
\text { American }\end{array}$ \\
\hline 32 & $24.5(18-35)$ & 9 & 23 & 14 & 10 & 7 & 1 \\
\hline
\end{tabular}


Table 2. List of megapools (MP) used in this study

\begin{tabular}{|c|c|c|c|c|}
\hline MP name & Source & Peptide/Epitope type & $\begin{array}{l}\text { Peptide } \\
\text { number }\end{array}$ & Reference \\
\hline NL63 & HCoV-NL63 & Predicted & 280 & $\begin{array}{l}\text { da Silva Antunes et al., JID } \\
\qquad(2021)^{26}\end{array}$ \\
\hline $229 \mathrm{E}$ & $\mathrm{HCoV}-229 \mathrm{E}$ & Predicted & 225 & $\begin{array}{l}\text { da Silva Antunes et al., JID } \\
\qquad(2021)^{26}\end{array}$ \\
\hline HKU1 & HCoV-HKU1 & Predicted & 320 & $\begin{array}{l}\text { da Silva Antunes et al., JID } \\
\qquad(2021)^{26}\end{array}$ \\
\hline OC43 & $\mathrm{HCoV}-\mathrm{OC} 43$ & Predicted & 294 & $\begin{array}{l}\text { da Silva Antunes et al., JID } \\
\qquad(2021)^{26}\end{array}$ \\
\hline SARS-CoV-2 & SARS-CoV-2 & Predicted & 474 & $\begin{array}{l}\text { da Silva Antunes et al., JID } \\
\qquad(2021)^{26}\end{array}$ \\
\hline $\mathrm{CMV}$ & $\mathrm{CMV}$ & Experimentally defined & 313 & $\operatorname{IEDB}^{55}$ \\
\hline EBV & EBV & Experimentally defined & 301 & $\operatorname{IEDB}^{55}$ \\
\hline Flu & Influenza A & Overlapping (HA) / Experimentally defined & 330 & $\operatorname{IEDB}^{55}$ \\
\hline RSV & RSV & Experimentally defined / Predicted & 216 & $\operatorname{IEDB}^{55}$ \\
\hline Rhinovirus & Rhinovirus & Experimentally defined & 136 & $\begin{array}{c}\text { Grifoni et al., } 2019 \text { Hum } \\
\text { Immunol }^{56}\end{array}$ \\
\hline VZV & VZV & $\begin{array}{l}\text { Glycoprotein E (gE) Experimentally } \\
\text { defined / Rest of genome (R) Predicted }\end{array}$ & 335 & Voic et al., J Virol $(2020)^{39}$ \\
\hline TT & $\begin{array}{l}\text { Clostridium } \\
\text { tetani }\end{array}$ & Experimentally defined & 125 & $\begin{array}{l}\text { da Silva Antunes et al., Plos } \\
\text { One }(2017)^{57}\end{array}$ \\
\hline PT & $\begin{array}{l}\text { Bordetella } \\
\text { pertussis }\end{array}$ & Experimentally defined & 132 & $\begin{array}{l}\text { da Silva Antunes et al., JIR } \\
\qquad(2020)^{58}\end{array}$ \\
\hline
\end{tabular}

